\title{
Flavour Physics in an SO(10) Grand Unified Model
}

\author{
Jennifer Girrbach* \\ Technische Universität München (TUM) \\ Institute of Advanced Study (IAS) \\ Excellence Cluster Universe \\ E-mail: jennifer.girrbach@ph.tum.de
}

Grand unified theories open the possibility to transfer the neutrino mixing matrix $U_{\text {PMNS }}$ to the quark sector. This is accomplished in a controlled way in a supersymmetric grand-unified model proposed by Chang, Masiero and Murayama (CMM model) where the atmospheric neutrino mixing angle induces large new $b \rightarrow s$ and $\tau \rightarrow \mu$ transitions. Relating the supersymmetric low-energy parameters to seven new parameters $a_{0}, m_{0}^{2}, m_{\tilde{g}}, D, \xi, \tan \beta$ and $\arg (\mu)$ of this $\mathrm{SO}(10)$ model, we perform a correlated study of several flavour-changing neutral current (FCNC) processes. The CMM model can serve as an alternative benchmark scenario to the popular constraint MSSM.

The 2011 Europhysics Conference on High Energy Physics, EPS-HEP 2011,

July 21-27, 2011

Grenoble, Rhône-Alpes, France

* Speaker. 


\section{Introduction}

Supersymmetric grand unified theories (SUSY GUTs) are popular extensions of the Standard Model (SM). The generic Minimal Supersymmetric Standard Model (MSSM) has (too) many sources of flavour and $\mathrm{CP}$ violation which reside in the soft breaking terms. Contrarily, in the minimal flavour violating (MFV) version of the MSSM large effects in the flavour sector can only appear in very few processes, such as $b \rightarrow s \gamma$. SUSY GUTs can lie somewhere in between. The unification of quarks and leptons into symmetry multiplets implies additional relations between SM parameters and correlation between the flavour mixing. Let's consider SU(5) multiplets:

$$
\overline{\mathbf{5}}_{1}=\left(\begin{array}{c}
d_{R}^{c} \\
d_{R}^{c} \\
d_{R}^{c} \\
e_{L} \\
-v_{e}
\end{array}\right), \quad \overline{\mathbf{5}}_{2}=\left(\begin{array}{c}
s_{R}^{c} \\
s_{R}^{c} \\
s_{R}^{c} \\
\mu_{L} \\
-v_{\mu}
\end{array}\right), \quad \overline{\mathbf{5}}_{3}=\left(\begin{array}{c}
b_{R}^{c} \\
b_{R}^{c} \\
b_{R}^{c} \\
\tau_{L} \\
-v_{\tau}
\end{array}\right) .
$$

If the PMNS matrix $U_{\mathrm{PMNS}}$ stems from a mixing of these 5-plets, then the corresponding mixing angles should also occur in the charged-lepton sector and right-handed down-quark sector. Especially the large atmospheric neutrino mixing angle $\theta_{23} \approx 45^{\circ}$ induces $b_{R} \rightarrow s_{R}$ and $\tau_{L} \rightarrow \mu_{L}$ transitions. Whereas mixing of right-handed quark fields in flavour space is unphysical it is not for the corresponding superfields due to the soft breaking terms. Consequently squark-gluino loops can induce $b_{R} \rightarrow s_{R}$ transitions. Further slepton-neutralino/sneutrino-chargino loops can induce $\tau \rightarrow \mu$ transitions at an observable level. This was the main idea of Moroi and Chang, Masiero and Murayama in [1, 2]. Similar and related works can be found e.g. in [3, 4]. In [5] we have performed a global analysis in the CMM model including an extensive renormalization group (RG) analysis to connect Planck-scale and low-energy parameters. In the next section we sketch the theoretical framework focusing on the flavour structure.

\section{The CMM model - a new benchmark scenario}

\subsection{Theory}

The idea of PMNS-like mixing of down-quark singlets and lepton doublets as discussed above is encoded in the following $\mathrm{SO}(10)$ superpotential:

$$
W_{Y}^{\mathrm{SO}(10)}=\frac{1}{2} \mathbf{1 6}_{i} Y_{1}^{i j} \mathbf{1 6}_{j} \mathbf{1 0}_{H}+\mathbf{1 6}_{i} Y_{2}^{i j} \mathbf{1 6}_{j} \frac{\mathbf{4 5}_{H} \mathbf{1 0}_{H}^{\prime}}{2 M_{\mathrm{Pl}}}+\mathbf{1 6}_{i} \mathrm{Y}_{N}^{i j} \mathbf{1 6}_{j} \frac{\overline{\mathbf{1 6}}_{H} \overline{\mathbf{1 6}}_{H}}{2 M_{\mathrm{Pl}}},
$$

where $M_{\mathrm{Pl}}$ is the Planck mass, $\mathbf{1 6}_{i}(i=1,2,3)$ is the $\mathrm{SO}(10)$ spinor representation (one matter field per generation) and $\mathbf{1 0}_{H}, \mathbf{1 0}_{H}^{\prime}, \mathbf{4 5}_{H}$ and $\overline{\mathbf{1 6}}_{H}$ are four Higgs superfields, where $\mathbf{1 0}_{H}$ contains the MSSM $H_{u}$ and $\mathbf{1 0}_{H}^{\prime}$ the MSSM $H_{d}$. One assumption of the CMM model is that $\mathrm{Y}_{1}$ and $\mathrm{Y}_{N}$ are simultaneously diagonalisable which can be achieved through a suitable flavour symmetry at $M_{\mathrm{Pl}}$. This flavour symmetry is broken by the second term in (2.1) with the consequence that the rotation matrix of the right-handed down-squarks is exactly $U_{\mathrm{PMNS}}$. SUSY is broken flavour blind at $M_{\mathrm{Pl}}$ implying universal soft- and trilinear terms. That is, the nonrenormalisable term $\propto \mathrm{Y}_{2}$ in 
the superpotential contains the whole flavour structure, its diagonalisation involves the PMNS and CKM matrices (up to rephasings). The symmetry breaking chain reads

$$
\mathrm{SO}(10) \stackrel{\left\langle 16_{H}\right\rangle,\left\langle\overline{16}_{H}\right\rangle}{\left\langle 45_{H}\right\rangle} \mathrm{SU}(5) \stackrel{\left\langle 45_{H}\right\rangle}{\longrightarrow} \mathrm{G}_{\mathrm{SM}} \stackrel{\left\langle 10_{H}\right\rangle,\left\langle 10_{H}^{\prime}\right\rangle}{\longrightarrow} \mathrm{SU}(3)_{C} \times \mathrm{U}(1)_{\mathrm{em}},
$$

which gives naturally small $\tan \beta$. Then $\mathrm{Y}_{1}$ gives masses to up-type fermions, $\mathrm{Y}_{2}$ to down-type fermions and $Y_{N}$ to right-handed Majorana neutrinos. We want to stress that flavour physics observables depend very weakly on the details of the Higgs potential which was not specified in the original paper [2]. But our results motivate further work on the Higgs potential.

The key ingredient for the flavour structure is the following: In a weak basis with diagonal up-type Yukawa matrix we have

$$
\mathrm{Y}_{d}=\mathrm{Y}_{\ell}^{\top}=V_{\mathrm{CKM}}^{\star}\left(\begin{array}{ccc}
y_{d} & 0 & 0 \\
0 & y_{s} & 0 \\
0 & 0 & y_{b}
\end{array}\right) U_{D}, \quad U_{D}=U_{\mathrm{PMNS}}^{*} \operatorname{diag}\left(1, e^{i \xi}, 1\right)
$$

and the right-handed down squark mass matrix at the low scale reads

$$
m_{\tilde{d}}^{2}\left(M_{Z}\right)=\operatorname{diag}\left(m_{\tilde{d}_{1}}^{2}, m_{\tilde{d}_{1}}^{2}, m_{\tilde{d}_{1}}^{2}\left(1-\Delta_{\tilde{d}}\right)\right),
$$

where $\Delta_{\tilde{d}} \in[0,1]$ defines the relative mass splitting between the $1^{\text {st }} / 2^{\text {nd }}$ and $3^{\text {rd }}$ down-squark generation. It is generated by RG effects of the top Yukawa coupling and can easily reach 0.4 . If we rotate to mass eigenstate basis and diagonalise $Y_{d}$ the neutrino mixing enters $m_{\tilde{D}}^{2}$ :

$$
m_{\tilde{D}}^{2}=U_{D} m_{\tilde{d}}^{2} U_{D}^{\dagger}=m_{\tilde{d}_{1}}^{2}\left(\begin{array}{ccc}
1 & 0 & 0 \\
0 & 1-\frac{1}{2} \Delta_{\tilde{d}} & -\frac{1}{2} \Delta_{\tilde{d}} e^{i \xi} \\
0 & -\frac{1}{2} \Delta_{\tilde{d}} e^{-i \xi} & 1-\frac{1}{2} \Delta_{\tilde{d}}
\end{array}\right)
$$

Consequently, the 23-entry $\propto \Delta_{\tilde{d}}$ is responsible for $\tilde{b}_{R}-\tilde{s}_{R}$-mixing and exactly here a new CP phase $\xi$ enters that affects $B_{s}-\bar{B}_{s}$ mixing. Note that there are zeros in the 12- and 13-entries, thus no effects in $K-\bar{K}$ and $B_{d}-\bar{B}_{d}$ mixing appear. This is due to the degeneracy of the first two squark generation and the assumed tribimaximal structure of $U_{\mathrm{PMNS}}$.

\subsection{Comparison with CMSSM/mSUGRA}

Only seven parameters of the CMM model are relevant for our analysis: the universal scalar soft mass $m_{0}$ and trilinear coupling $a_{0}$ at the Planck scale, the gluino mass $m_{\tilde{g}}$, the $D$-term mass splitting $D$, the phase of $\mu$, the phase $\xi$ and $\tan \beta$ (but $2.7 \lesssim \tan \beta \lesssim 10$ ). We did a comprehensive RG evolution to relate Planck-scale inputs to a set of low-energy inputs: the masses of $\tilde{u}_{R}$ and $\tilde{d}_{R}$ of the first generations $m_{\tilde{u}_{1}}, m_{\tilde{d}_{1}}$, the 11-element of the trilinear coupling of the down squarks $a_{1}^{d}$, $m_{\tilde{g}}, \arg \mu, \xi$ and $\tan \beta$. We evolve these parameters twice from $M_{\text {ew }}$ to $M_{\text {Planck }}$ and back to $M_{\text {ew }}$ to find all particle masses and MSSM couplings.

The minimal supergravity (mSUGRA) scenario or its popular variant, the constraint MSSM (CMSSM), has - similar to the CMM model - only a few input parameters. But the philosophy is somewhat different: the CMSSM minimises flavour violation in an ad-hoc way and assumes flavour 


\begin{tabular}{|c|c|c|}
\hline generic MSSM & mSUGRA/CMSSM & CMM model \\
\hline \hline$\approx 120$ parameters & 4 parameters \& 1 sign & 7 input parameters \\
\hline SUSY flavour \& CP problem & $\begin{array}{c}\text { minimize flavour } \\
\text { violation ad-hoc }\end{array}$ & clear flavour structure \\
\hline no universality & universality at $M_{\mathrm{GUT}}$ & $\begin{array}{c}\text { universality at } M_{\mathrm{Pl}} \\
\text { but broken at } M_{\mathrm{GUT}}\end{array}$ \\
\hline \multicolumn{2}{|c|}{ quarks \& leptons unrelated } & quark-lepton-interplay \\
\hline $\begin{array}{c}\text { Problem: suppress large } \\
\text { effects elsewhere }\end{array}$ & $\begin{array}{c}\text { cannot explain current } \\
\left.\text { flavour data (e.g. } \phi_{s}\right)\end{array}$ & $\begin{array}{c}\text { can fit } \phi_{s} \text { and small } \\
\text { effects in 1st/2nd gen. }\end{array}$ \\
\hline
\end{tabular}

Table 1: Comparison between the generic MSSM, mSUGRA/CMSSM and the CMM model

universality at the GUT scale with quark and lepton flavour structures being unrelated. However the CMM model has a clear flavour structure different from MFV and universality is already broken at $M_{\mathrm{GUT}}$. Furthermore due to this free phase $\xi$, one can fit the $B_{s}-\bar{B}_{s}$ mixing phase $\phi_{s}$ to the data. Also the particle spectrum is quite different between the CMSSM and the CMM model (mainly due to the large mass splitting $\Delta_{\tilde{d}}$ ). This comparison is summarized in tab. 1 . Hence the CMM model could serve as a new benchmark model: it is well-motivated, has only seven input parameters and it is a very predictive alternative to the well-studied CMSSM.

\subsection{Phenomenology}

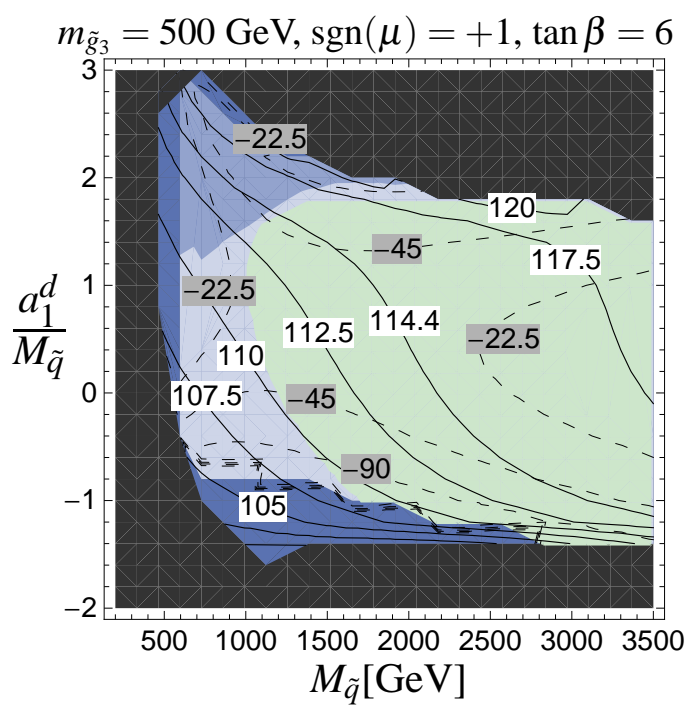

- black: $m_{\tilde{f}}^{2}<0$, unstable vacuum

- dark blue: excluded by $B_{s}-\bar{B}_{s}$

- medium blue: excluded by $b \rightarrow s \gamma$

- light blue: excluded by $\tau \rightarrow \mu \gamma$

- green: compatible with $B_{s}-\bar{B}_{s}, b \rightarrow s \gamma$, $\tau \rightarrow \mu \gamma$

- Higgs mass in GeV: solid line with white labels

- $\phi_{s}$ with maximal possible $\left|\phi_{s}\right|$ in degrees: dashed line with gray labels

Figure 1: Correlation of FCNC processes as a function of $M_{\tilde{q}}\left(M_{Z}\right)$ (degenerate squark mass of first two generations) and $a_{1}^{d}\left(M_{Z}\right) / M_{\tilde{q}}\left(M_{Z}\right)$ for $m_{\tilde{g}}\left(M_{Z}\right)=500 \mathrm{GeV}$ and $\operatorname{sgn}(\mu)=+1$ with $\tan \beta=6$.

We did a global analysis of flavour observables where we expected large CMM effects, namely $B_{s}-\bar{B}_{s}$ mixing, $b \rightarrow s \gamma$ and $\tau \rightarrow \mu \gamma$. Moreover we included vacuum stability bounds, lower bounds on sparticle masses and the mass of the lightest Higgs boson. The result is shown in fig. 1. The flavour effects are proportional to $\Delta_{\tilde{d}}$ and maximized for small $\tan \beta$. However, the Higgs mass constraint excludes too small values for $\tan \beta$. With $\xi$ we can accommodate a large $\phi_{s}$ while simul- 
taneously fulfilling all other experimental constraints. The branching ratio $B R\left(B_{s} \rightarrow \mu^{+} \mu^{-}\right)$does not get large CMM effects because $\tan \beta$ is small. Realistic GUTs involve dimension-5 Yukawa terms to fix the relation $\mathrm{Y}_{d}=\mathrm{Y}_{\ell}^{\top}$ for the $1^{\text {st }}$ and $2^{\text {nd }}$ generation. Consequently we do not only get $b_{R} \rightarrow s_{R}$ but also $b_{R} \rightarrow d_{R}$ and $d_{R} \rightarrow s_{R}$ transition. This has been worked out in [6] and is strongly constraint by $K-\bar{K}$ mixing. Similar constraints can be found from $\mu \rightarrow e \gamma$ [7].

\section{Conclusion}

SUSY GUTs are theoretical well-motivated scenarios with correlations between hadronic and leptonic observables. If large $\mathrm{CP}$ violation in $B_{s}-\bar{B}_{s}$ mixing is confirmed we need physics beyond the CMSSM and mSUGRA. We advertise the CMM model where the large atmospheric neutrino mixing angle $\theta_{23} \approx 45^{\circ}$ induces $b-s$ - and $\tau-\mu$-transitions as an alternative benchmark scenario. We did an extensive RG analysis of the CMM model relating several observables ( $B_{s}-\bar{B}_{s}$ mixing, $b \rightarrow s \gamma, \tau \rightarrow \mu \gamma, m_{h}$, vacuum stability bounds and lower bounds on sparticle masses) to seven new input parameters beyond those of the SM. Due to a free phase $\xi$ we can adjust CP violation in $B_{s}-\bar{B}_{s}$ mixing while at the same time getting only minor effects in $2 \rightarrow 1$ and $3 \rightarrow 1$ transitions.

\section{Acknowledgments}

I thank the organisers for the opportunity to give this talk and my collaborators S. Jäger, M. Knopf, W. Martens, U. Nierste, C. Scherrer and S. Wiesenfeldt for an enjoyable collaboration. I thank U. Nierste for proofreading this manuscript. My work on the presented topic was supported by the Studienstiftung des deutschen Volkes and I acknowledge financial support by the DFG cluster of excellence "Origin and Structure of the Universe".

\section{References}

[1] T. Moroi, JHEP 0003 (2000) 019. [hep-ph/0002208]; Phys. Lett. B 493 (2000) 366. [hep-ph/0007328].

[2] D. Chang, A. Masiero and H. Murayama, Phys. Rev. D 67 (2003) 075013, [hep-ph/0205111].

[3] R. Barbieri, L. J. Hall and A. Strumia, Nucl. Phys. B 449 (1995) 437. [hep-ph/9504373]; Nucl. Phys. B 445 (1995) 219. [hep-ph/9501334].

[4] R. Harnik, D. T. Larson, H. Murayama and A. Pierce, Phys. Rev. D 69 (2004) 094024. [hep-ph/9501334].

[5] J. Girrbach, S. Jager, M. Knopf, W. Martens, U. Nierste, C. Scherrer and S. Wiesenfeldt, JHEP 1106 (2011) 044 [Erratum-ibid. 1107 (2011) 001] [arXiv:1101.6047 [hep-ph]].

[6] S. Trine, S. Westhoff, S. Wiesenfeldt, JHEP 0908 (2009) 002. [arXiv:0904.0378 [hep-ph]].

[7] J. Girrbach, S. Mertens, U. Nierste, S. Wiesenfeldt, JHEP 1005 (2010) 026. [arXiv:0910.2663 [hep-ph]].

P. Ko, J. -h. Park, M. Yamaguchi, JHEP 0811 (2008) 051. [arXiv:0809.2784 [hep-ph]]. 